\title{
DNA polymorphism in recombining and non-recombing mating-type-specific loci of the smut fungus Microbotryum
}

\author{
AA Votintseva and DA Filatov \\ Department of Plant Sciences, University of Oxford, Oxford, UK
}

\begin{abstract}
The population-genetic processes leading to the genetic degeneration of non-recombining regions have mainly been studied in animal and plant sex chromosomes. Here, we report population genetic analysis of the processes in the non-recombining mating-type-specific regions of the smut fungus Microbotryum violaceum. $M$. violaceum has A1 and A2 mating types, determined by mating-type-specific 'sex chromosomes' that contain 1-2 Mb long non-recombining regions. If genetic degeneration were occurring, then one would expect reduced DNA polymorphism in the non-recombining regions of this fungus. The analysis of DNA diversity among 19 M. violaceum strains, collected across Europe from Silene latifolia flowers, revealed that (i) DNA polymorphism is relatively low in all 20 studied loci $(\pi \sim 0.15 \%)$, (ii) it is not significantly different between the two mating-type-specific
\end{abstract}

chromosomes nor between the non-recombining and recombining regions, (iii) there is substantial population structure in $M$. violaceum populations, which resembles that of its host species, S. latifolia, and (iv) there is significant linkage disequilibrium, suggesting that widespread selfing in this species results in a reduction of the effective recombination rate across the genome. We hypothesise that selfing-related reduction of recombination across the $M$. violaceum genome negates the difference in the level of DNA polymorphism between the recombining and non-recombining regions, and may possibly lead to similar levels of genetic degeneration in the mating-type-specific regions of the non-recombining 'sex chromosomes' and elsewhere in the genome.

Heredity (2011) 106, 936-944; doi:10.1038/hdy.2010.140; published online 17 November 2010

Keywords: Microbotryum; recombination; selfing; DNA polymorphism

\section{Introduction}

Recombination is a major determinant in genome evolution (Gaut et al., 2007; Larracuente et al., 2008). Regions that lack recombination tend to lose functional genes and accumulate junk DNA because the evolutionary fates of mutations in non-recombining regions are not independent and natural selection cannot effectively eliminate deleterious and fix advantageous mutations (Hill and Robertson, 1966; Felsenstein, 1974). The best studied cases of such genetic degeneration include animal and plant sex chromosomes (Charlesworth and Charlesworth, 2005). Despite their independent evolution in different groups of animals and plants (Bull, 1983), sex chromosomes have similar properties: recombination is restricted between the $X$ and $Y$ chromosomes, and the male-specific non-recombining $\mathrm{Y}$ chromosome exhibits genetic degeneration-the loss of functional genes and accumulation of repetitive DNA. Similar processes are expected to occur in other nonrecombining regions, such as plant self-incompatibility loci and fungal mating-type loci. Indeed, it has been suggested that mating-type-determining regions of

Correspondence: Dr DA Filatov, Department of Plant Sciences, University of Oxford, South Parks Road, Oxford OX1 3RB, UK.

E-mail: dmitry.filatov@plants.ox.ac.uk

Received 6 December 2009; revised 21 July 2010; accepted 6 September 2010; published online 17 November 2010 fungi, such as Cryptococcus neoformans (Lengeler et al., 2002), Neurospora tetrasperma (Menkis et al., 2008) and Microbotryum violaceum (Hood, 2002; Votintseva and Filatov, 2009), represent fungal 'sex chromosomes'. However, at present there is no solid evidence for genetic degeneration in fungal mating-type-determining regions. For example, the mating-type locus of the fungal pathogen Cryptococcus neoformans contains $\sim 20$ genes in about $100 \mathrm{~kb}$ of non-recombining DNA and does not show any obvious signs of genetic degeneration (Lengeler et al., 2002; Fraser et al., 2004). Here, we focus on the analysis of population-genetic processes dominating evolution in the non-recombining mating-type-specific regions of the smut fungus $M$. violaceum.

Microbotryum is a basidiomycete that causes anthersmut disease in many species in the plant family Caryophyllaceae. Until recently, the same species name, $M$. violaceum, has been used for strains parasitising different host species. However, multiple sources of evidence (sequence divergence, teliospore colour, crossmating experiments) support the view that strains on different hosts represent separate species (Le Gac et al., 2007; De Vienne et al., 2009). The systematics of the genus is being revised to assign species names to strains with different host specificities. In particular, the Microbotryum strains from Silene latifolia analysed below are now called Microbotryum lychnidis-dioicae, but the classification is not yet settled (Denchev et al., 2009), and so here, we will use the traditional species complex name $M$. violaceum to 
refer to strains collected from S. latifolia. For strains collected from other host species, we will specify the species name of the host plant.

The life cycle of $M$. violaceum has been reviewed recently (Giraud et al., 2008). Dispersal of the fungus occurs via teliospores that form in the anthers of infected plants and are spread by pollinators. Interestingly, in female plants of dioecious Silene, the smut can cause masculinisation of the phenotype and the formation of anther-like structures (Ruddat et al.., 1991). Teliospores undergo meiosis on the host plant, and the resulting haploid cells bud off sporidia that eventually conjugate to form infectious dikaryons. Dikaryotic hyphae biotrophically proliferate through host plant tissues. The fungus has a bipolar heterothallic breeding system, and conjugation occurs only between sporidia of opposite mating types. The two mating types, A1 and A2, are determined by a mating-type-specific region, which is similar to the $\mathrm{Y}$ chromosomes of animals and plants, as recombination is suppressed in a large region around the mating-type-determining genes (Hood, 2002; Votintseva and Filatov, 2009).

The A1 and A2 mating-type-specific chromosomes of $M$. violaceum are distinguishable by size in pulse-gel electrophoresis (Hood, 2002), and multiple fragments from the A1, A2 and other chromosomes have been isolated (Hood et al., 2004). The analysis of these fragments suggested a lower gene density on the mating-type-specific chromosomes, compared with the rest of the genome in this species (Hood et al., 2004). However, a lower gene density may be due to the addition of junk DNA to the mating-type-specific chromosomes rather than the loss of functional genes. Recent segregation analysis of the mating-type specificity of 86 fragments isolated from the A1 and A2 chromosomes revealed that only about a quarter are located in the non-recombining mating-type-specific regions, whereas the rest mapped to recombining regions and are not expected to undergo genetic degeneration because of a lack of recombination (Votintseva and Filatov, 2009). Based on this analysis, the size of the nonrecombining regions was estimated to be about $1 \mathrm{Mb}$ long on each of the mating-type-specific chromosomes; however, this estimate is very approximate. Thus, it remains unclear whether or not the non-recombining mating-type-specific chromosomes in $M$. violaceum are undergoing genetic degeneration via gene loss, as is typical for $\mathrm{Y}$ chromosomes in animals and plants.

Comparisons of DNA polymorphism in recombining and non-recombining regions provide a way to test whether the population-genetic processes causing genetic degeneration are acting in the region of interest. The complete linkage of genes in non-recombining regions, such as $\mathrm{Y}$ chromosomes, makes the evolutionary fates of mutations in such regions non-independent, which leads to genetic hitchhiking (the fixation of deleterious mutations due to linkage to favourable mutations spreading in the population, (Maynard Smith and Haigh, 1974; Rice, 1987)) and background selection (selection against deleterious mutations at linked genes, resulting in a reduced effective population size and stochastic accumulation of mildly deleterious mutations, (Charlesworth et al., 1993, 1995)). These processes reduce the effective population size and lead to the accumulation of deleterious mutations and a gradual genetic degeneration.
Indeed, multiple studies have reported reduced genetic diversity on $\mathrm{Y}$ chromosomes, compared with the $X$-linked or autosomal genes, in animal and plant species (Filatov et al., 2000; Yi and Charlesworth, 2000).

Another factor affecting genetic diversity throughout $M$. violaceum genome is its tendency to mate between the products of the same meiosis (Hood and Antonovics, 2004). This leads to high degree of selfing, which is expected to reduce diversity throughout the genome. Indeed, the analysis of microsatellite diversity revealed high homozygosity (Bucheli et al., 2001; Giraud, 2004; Granberg et al., 2008). However, DNA diversity in $M$. violaceum populations has not been studied. Here, we analyse DNA polymorphism in a set of mating-typespecific loci in $M$. violaceum and compare it with DNA polymorphism in loci not linked to the mating-type locus. If non-recombining mating-type-specific regions do undergo genetic degeneration in M. violaceum, similar to the $\mathrm{Y}$ chromosomes of diploid species, then loci in these regions are expected to have reduced genetic diversity compared with non-mating-type-specific loci; however, it is not clear whether the reduction of DNA diversity in the non-recombining regions is detectable if the overall DNA diversity is reduced because of selfing.

\section{Materials and methods}

\section{Samples}

The samples of $M$. violaceum used in this study were collected across Europe and are listed in Table 1. Teliospores were collected from flowers of S. latifolia plants, and cultures were grown in petri dishes on $3.6 \%$ potato dextrose agar medium. DNA was extracted from these mixed mating-type cultures using Invitrogen Charge Switch magnetic beads-based DNA extraction

Table $1 M$. violaceum samples used in the study

\begin{tabular}{llll}
\hline ID & Host plant & Location & Country \\
\hline Sl-769 & S. latifolia & Galicia & Spain \\
Sl-920 & S. latifolia & Valencia & Spain \\
Sl-562 & S. latifolia & nr Krakow & Poland \\
Sl-563 & S. latifolia & nr Krakow & Poland \\
Sl-405 & S. latifolia & nr Stockholm & Sweden \\
Sl-406 & S. latifolia & nr Stockholm & Sweden \\
Sl-432 & S. latifolia & nr Stockholm & Sweden \\
Sl-433 & S. latifolia & nr Stockholm & Sweden \\
Sl-1088 & S. latifolia & nr Lausanne & Switzerland \\
Sl-1089 & S. latifolia & nr Lausanne & Switzerland \\
Sl-127 & S. latifolia & Pyrenees & France \\
Sl-320 & S. latifolia & Central & France \\
Sl-321 & S. latifolia & North & France \\
Sl-1099 & S. latifolia & nr Guéret & France \\
Sl-144 & S. latifolia & nr Chernobyl & Ukraine \\
Sl-1069 & S. latifolia & Cotswolds & England \\
Sl-1103 & S. latifolia & nr Woodstock & England \\
Sl-1075 & S. latifolia & Sardinia & Italy \\
Sl-1076 & S. latifolia & Sardinia & Italy \\
Sd-1060 & S. dioica & East & Scotland \\
Sd-1066 & S. dioica & West & Scotland \\
Sn-87 & S. nutans & Alps & France \\
Sv-1 & S. vulgaris & nr La Sage & Switzerland \\
Lfc-109 & L. flos-cuculi & Alps & France \\
\hline Albreviand & S &
\end{tabular}

Abbreviations: L. flos-cuculi, Lychnis flos-cuculi; M. violaceum, Microbotryum violaceum; $n r$, near; S. dioica, Silene dioica; S. latifolia, Silene latifolia; S. nutans, Silene nutans; S. vulgaris, Silene vulgaris. 
DNA polymorphism in a smut fungus AA Votintseva and DA Filatov

kit (Invitrogen Ltd, Paisley, UK). Two samples of $M$. violaceum were also collected from Silene dioica plants, which were used as an outgroup, mainly for HKA (Hudson, Kreitman \& Aguade) analysis (Hudson et al., 1987), see below. We also attempted to obtain more divergent outgroup sequences from $M$. violaceum strains collected from S. vulgaris, S. nutans and Lychnis flos-cuculi. Total nucleotide divergence between these outgroups and the sequences from $M$. violaceum strains collected from S. latifolia exceeded 5\%, and in some cases, we could not amplify the loci from these more diverged strains. Using these more diverged sequences changes neither the outcome of the HKA test nor the conclusions of the work; hence, throughout the paper, we use $M$. violaceum from S. dioica as an outgroup, as it is available for all loci in this study.

\section{Loci}

Using pulse-gel electrophoresis, Hood et al. (2004) isolated and sequenced random fragments from the A1, A2 and other chromosomes of M. violaceum. In an earlier study, we demonstrated that only a fraction of loci isolated from the A1 and A2 chromosomes are linked to mating type, whereas the rest are located in recombining regions and are not mating-type-specific (Votintseva and Filatov, 2009). In this study, we use seven loci that are located in the non-recombining region specific to the A1 mating type and five loci specific to the A2 mating type. We also used eight non-mating-type-specific loci from recombining regions (Table 2) for comparisons with the loci in the non-recombining regions. Two of the matingtype-specific loci corresponded to pheromone receptor genes from the A1 and A2 chromosomes (Yockteng et al.,
2007), whereas the rest of the recombining and nonrecombining loci were anonymous fragments originally isolated by Hood et al. (2004).

\section{DNA sequencing}

PCR primers for amplification and sequencing are listed in Table 2. PCR amplification was conducted using Bioline BioMixRed PCR kits (BioLine inc., Taunton, MA, USA). The PCR fragments were checked by standard agarose gel electrophoresis and purified using Qiagen PCR purification or Gel extraction kits (Qiagen Ltd, Crawley, UK). Direct DNA sequencing of PCR-amplified fragments was conducted using ABI BigDye v3.1 on an ABI 3730 automated DNA sequencer (Applied Biosystems, Foster City, CA, USA). Sequence chromatograms were corrected, and contigs were assembled and checked using ProSeq3 (Filatov, 2009). The sequences were submitted to GenBank under accession numbers HM176794-HM177244.

Direct sequencing of PCR products from mating-typespecific loci generates haploid data, whereas sequencing of the non-mating-type-specific loci generates diploid unphased sequences. Visual inspection of the sequencing chromatograms revealed that only two loci in two individuals were heterozygous for several nucleotide polymorphisms, whereas the rest were completely homozygous, which simplified the reconstruction of haplotypes for non-mating-type-specific loci. Haplotypes for these sequences were reconstructed using fastPhase (Scheet and Stephens, 2006). Alignments of the resulting sequences were loaded into a ProSeq3 database, allowing sequences to be linked to the individuals from which they were obtained. Such linking makes the handling and analysis of multigenic DNA polymorphism data sets more convenient (Filatov,

Table 2 Loci used in this study

\begin{tabular}{|c|c|c|c|c|}
\hline \multirow[t]{2}{*}{$\mathrm{N}$} & \multirow[t]{2}{*}{ Loci } & \multicolumn{2}{|c|}{ PCR primers } & \multirow[t]{2}{*}{$\mathrm{Tm},{ }^{\circ} \mathrm{C}^{\mathrm{a}}$} \\
\hline & & Forward primers & Reverse primers & \\
\hline \multicolumn{5}{|c|}{ Non-recombining A1 mating-type specific } \\
\hline 1 & A1-pr & A1-prF TGGCATCCCTCAATGTTTCC & A1-prR CACCTTTTGATGAGAGGCCG & $54.6 / 54.6$ \\
\hline 2 & A1-077 & A1-077f CCCTCGACGATGTTCTTCTTG & A1-077r CCAGTCGAATATTTGGAACCG & $53.8 / 53.7$ \\
\hline 3 & A1-116 & A1-116f TGAGTGATGGTCGACAGGTCA & A1-116r TGACATCAGCTGAGTTCCCAGT & $53.5 / 53.6$ \\
\hline 4 & A1-202 & A1-202f ACAGGTCATTCACAACGGCT & A1-202r TCCTCGACCGTATTTATCGTG & $51.8 / 51.9$ \\
\hline 5 & A1-210 & A1-210f GGAATGCAGTGGGTCATGGT & A1-210r CGTTGCGATGACGAGGTATCT & $54.3 / 54.2$ \\
\hline 6 & A1-276 & A1-276f AGAACAGAGGCCAAGGGAGC & A1-276r CCTGAACAACСCСАТСТАССС & $54.8 / 54.7$ \\
\hline 7 & A1-281 & A1-281f ACATGTTCGTAGGCGATTCG & A1-281r GTAGAGTAAGATGGCGGCCG & $52.7 / 53.9$ \\
\hline \multicolumn{5}{|c|}{ Non-recombining A2 mating-type specific } \\
\hline 8 & A2-pr & A2-prF TGACGAGAGCATTCCTACCG & A2prR GAAGCGGAACTTGCCTTTCT & $52.7 / 52.8$ \\
\hline 9 & A2-400 & A2-400f TGGATGAGATGAAGTTGCGC & A2-400r CATACATTTTGGCGGGTTCA & $53.5 / 53.3$ \\
\hline 10 & A2-441 & A2-441f GNCTTGAAGCGAAGGCAAAC & A2-441r CGAAACTTACGGCTGCCATAG & $54.1 / 54.2$ \\
\hline 11 & A2-500 & A2-500f GAAGAGGGGGTTTTGACGAC & A2-500r CCCAACATGACCTCTTTCGA & $52.5 / 52.3$ \\
\hline 12 & A2-530 & A2-530f CAAGATTGAGGATAGAAGATGGATG & A2-530r GAAGTATTCGAGGTTCTTGTGGTT & $53.0 / 52.6$ \\
\hline \multicolumn{5}{|c|}{ Recombining } \\
\hline 13 & paA1-134 & A1-134f GGGCTTCGGAAGAAATGATC & A1-134r TCTТССТТСССТCAGATGGC & $53.1 / 53.0$ \\
\hline 14 & paA1-211 & A1-211f ACAGATCTCACAAAGTCGCAGA & A1-211r CACAGTGGGCTATATTTACACAAAT & $53.2 / 53.2$ \\
\hline 15 & paA2-433 & A2-433f GTCTACCGAGTCAGACTGCACA & A2-433r GGATCTATGCAGCCTTCGAC & $53.5 / 53.4$ \\
\hline 16 & paA2-461 & A2-461f CAGAAATTGGAAGCGATTGA & A2-461r ATTCCTTGCAAGTCTCGACC & $53.3 / 53.2$ \\
\hline 17 & paA2-547 & A2-547f TGGCTGCTTCTTTATGACCA & A2-547r CAAACAATTTCAATCGATGCAG & $50.9 / 52.5$ \\
\hline 18 & Aut-932 & Aut-932f ATTCGACTGCTCCСАСТCTG & Aut-932r AGGAGGAGAAAGTCGATCGC & $51.6 / 52.5$ \\
\hline 19 & Aut-935 & Aut-935f TGTGGTCAGTAGAGAATGGCG & Aut-935r TCATCGTTGATCCGACCTGT & $52.7 / 52.5$ \\
\hline 20 & Aut-948 & Aut-948f GCTTGTTGTCAATCATTCATCA & Aut-948r TATGCTAATGCGGGAACAAG & $50.1 / 50.6$ \\
\hline
\end{tabular}

Abbreviation: A1-pr and A2-pr, A1- and A2-pheromone receptor genes.

aAnnealing temperature for each primer; PCR was conducted at the Tm intermediate for the two primers. 

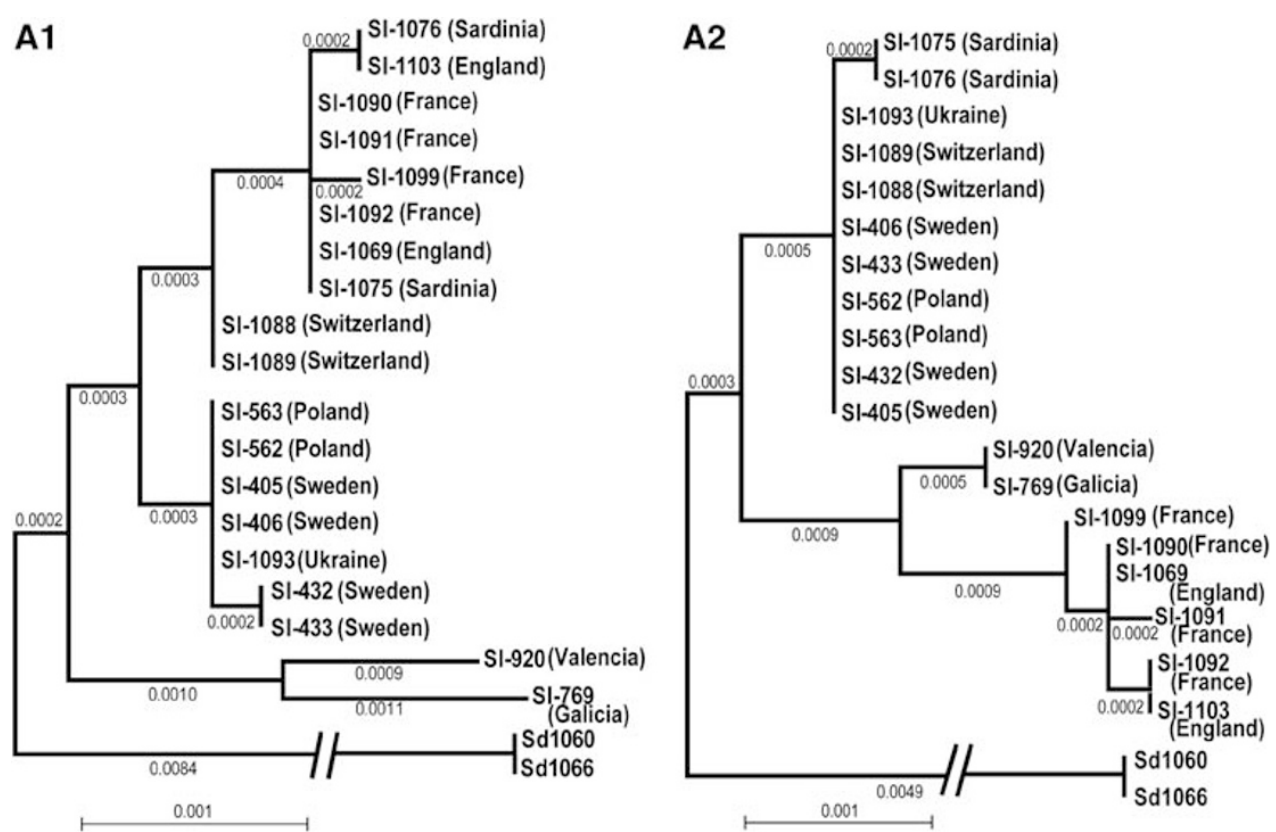

Figure 1 Neighbour-joining phylogenies of M. violaceum constructed from concatenated A1- and A2-linked genes. The branch lengths are proportional to the maximum-composite likelihood distance from MEGA4 (Tamura et al., 2007).

2009), as any action is applied once to all individuals rather than 20 times to each of the 20 alignments in the data set.

\section{DNA polymorphism analyses}

Neighbour-joining trees for concatenated non-recombining regions (Figure 1) were reconstructed with MEGA4 (Tamura et al., 2007). The basic descriptive statistics of DNA polymorphism, average number of differences pernucleotide, $\pi$ (Tajima, 1983), Watterson's $\theta$ (Watterson, 1975) and Tajima's D (Tajima, 1989) were calculated using ProSeq3 (Filatov, 2009). The HKA test (Hudson et al., 1987) was conducted using Jody Hey's HKA programme (http:/ / genfaculty.rutgers.edu/hey/software) on concatenations of all A1-linked, A2-linked and loci unlinked to mating type. Linkage disequilibrium was measured using the $D^{\prime}$ statistic (Lewontin, 1964) and its significance was assessed with $\chi^{2}$, as implemented in DnaSP5 (Librado and Rozas, 2009). The multilocus LD statistic ZnS (Kelly, 1997) was calculated using ProSeq3. The minimum number of recombination events, $R_{\min }$ (Hudson and Kaplan, 1985) and the population recombination rate, $R$ (Hudson, 1987) were estimated using DnaSP5. Bayesian clustering analysis of recombining loci was conducted with Structure2 software (Pritchard et al., 2000).

The power analysis to detect reduction of DNA polymorphism in the non-recombining regions, compared with recombining loci was conducted using coalescent simulations (Hudson, 2002) in two-deme model with no migration. In the simulation, one sequence corresponding to the outgroup was sampled from one deme, whereas the samples corresponding to the number of individuals sequenced from $M$. violaceum from S. latifolia plants were sampled from the other deme. The simulation parameters were adjusted to fit the observed level of polymorphism and divergence from the outgroup. While simulating non-recombining loci, we reduced polymorphism 4- and 10-fold (2- and 5-fold after adjustment for 2-fold ploidy difference), compared with recombining loci, whereas keeping divergence from the outgroup unchanged. The simulated 10000 data sets were run through J Hey's HKA programme using Perl script and the significance of rejecting neutrality was recorded.

\section{Results and discussion}

\section{DNA polymorphism}

To estimate DNA polymorphism in the non-recombining mating-type-specific regions, we sequenced seven A1specific and five A2-specific loci in $19 \mathrm{M}$. violaceum strains collected from $S$. latifolia populations across Europe (Tables 1 and 2). The absence of recombination in these loci was checked in a previously described genetic cross (Votintseva and Filatov, 2009). To compare the diversity in recombining and non-recombining regions, we also sequenced eight non-sex-specific loci in the same sample of $19 \mathrm{M}$. violaceum strains. Three of these loci were originally isolated by Hood et al., (2004) from chromosomes other than the $\mathrm{A} 1$ and $\mathrm{A} 2$, and five of the non-mating-type-specific loci come from fragments originally isolated from the A1 or A2 pulse-gel bands (Hood et al., 2004); however, they amplify from both mating types, and there is no divergence between the sequences of the fragments amplified from the A1 and A2 cultures (Votintseva and Filatov, 2009). We refer to all these fragments as 'recombining loci'.

The same regions were also sequenced from two outgroup samples of $M$. violaceum that parasitise $S$. dioica. DNA divergence between the strains parasitising $S$. latifolia and $S$. dioica was much higher than the polymorphism between the strains from $S$. latifolia (Figure 1). This supports the view that $M$. violaceum strains living on different Silene species are in fact 
Table 3 DNA polymorphism in non-recombining mating-typespecific (A1 and A2), and recombining genes for the entire sample of $M$. violaceum from S. latifolia plants

\begin{tabular}{llllllllll}
\hline & $\mathrm{N}^{\mathrm{a}}$ & $L^{\mathrm{b}}$ & $S^{\mathrm{c}}$ & $\begin{array}{c}\pi \\
(\%)^{\mathrm{d}}\end{array}$ & $\begin{array}{l}\text { s.d. } \\
(\pi)^{\mathrm{e}}\end{array}$ & Taj $^{\mathrm{f}}$ & $Z_{n S^{\mathrm{g}}}$ & $\mathrm{R}^{\mathrm{h}}$ & $\mathrm{R}_{\min }{ }^{\mathrm{i}}$ \\
\hline $\mathrm{A} 1$ & 19 & 4652 & 23 & 0.11 & 0.071 & -0.828 & 0.2738 & 0.0002 & 0 \\
$\mathrm{~A} 2$ & 19 & 3136 & 16 & 0.2 & 0.07 & 1.352 & 0.4168 & 0.0006 & 0 \\
$\mathrm{~A} 1+\mathrm{A} 2$ & 19 & 7791 & 39 & 0.15 & 0.071 & 0.088 & 0.24 & 0.0007 & 1 \\
Recombining & 38 & 5835 & 53 & 0.22 & 0.105 & 0.0228 & 0.1078 & 0.0066 & 9 \\
\hline
\end{tabular}

Abbreviations: M. violaceum, Microbotryum violaceum; S. latifolia, Silene latifolia.

${ }^{\mathrm{a}}$ Sample size.

${ }^{\mathrm{b}}$ Number of positions analysed (after excluding indels).

${ }^{\mathrm{c}}$ Number of polymorphic sites.

${ }^{\mathrm{d}}$ Average heterozygosity (per 100 sites).

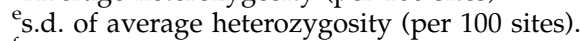

'Tajima's $D$ (Tajima, 1989).

gMultilocus linkage disequilibrium statistic ZnS (Kelly, 1997).

hPer-nucleotide population recombination rate (Hudson, 1987).

${ }^{\mathrm{i}}$ Minimal number of recombination events (Hudson and Kaplan, 1985).

separate species (Le Gac et al., 2007) and that the M. violaceum samples collected from $S$. latifolia belong to the same biological species, whereas $M$. violaceum from different host species (for example, $S$. dioica) belong to different species.

The total alignment length was $14044 \mathrm{bp}$, with $4896 \mathrm{bp}$ in the concatenated A1-linked loci, $3150 \mathrm{bp}$ in the A2linked loci and 5998bp in the non-mating-type-specific loci. The analysis of these alignments identified 23, 16 and 53 single-nucleotide polymorphisms between M. violaceum strains for the A1-, A2- and non-matingtype-specific loci, respectively (Table 3). As most loci analysed in this paper originated as random fragments isolated from mating-type-specific chromosomes, no information with regard to coding regions is available; thus, we do not distinguish between silent and non-silent sites, and report DNA polymorphism and divergence for all sites only. Average heterozygosity $(\pi)$ per 100 sites in the A1- and A2-linked non-recombining regions was relatively low, 0.11 and $0.20 \%$, respectively. Polymorphism in the recombining regions was similar, $0.22 \%$, providing no evidence that the lack of recombination in the mating-type-specific regions has led to a reduction in DNA polymorphism in M. violaceum (Table 3 ). Indeed, an HKA test that takes into account the twice lower ploidy for mating-type-specific loci as well as possible differences in mutation rates did not reject the null hypothesis $(P=0.887)$ that the differences in DNA polymorphism between the compared loci are due to chance. Coalescent simulations (see methods) demonstrate that with our data set we have 48 and $73 \%$ power to reject neutrality with two- and five-fold (after adjusting for ploidy difference) reduction of polymorphism in the nonrecombining region. Thus, the non-significant HKA for our data set suggests that if there is any reduction of DNA diversity in the non-recombining region of $M$. violaceum genome, such reduction is very modest.

A recurrent statement in fungal literature is that genetic diversity around the mating-type loci is expected to be higher than elsewhere in the genome (Glass et al., 1988; Zambino et al., 1997; James et al., 2004). This is only the case if the divergence between the mating-type-specific alleles is included in the estimate of polymorphism.
As mating-type-specific regions do not recombine, they gradually diverge from each other, which inflates overall genetic diversity in the region. A similar effect can be seen around self-incompatibility loci in plants and close to any locus under balancing selection (Uyenoyama, 2005; Charlesworth, 2006). However, this inflation of genetic diversity occurs only between the alleles and does not affect diversity within the allelic classes. In the case of $M$. violaceum, there are two allelic classes in the mating-type-specific region, A1- and A2specific alleles. If one includes both A1- and A2-specific alleles in the calculation of genetic diversity, it will be elevated around the mating-type locus (because A1 and A2 are diverged from each other), compared with the rest of the genome. On the other hand, taking into account only A1- or only A2-specific alleles, as was the case in our calculation of DNA diversity in Microbotryum, genetic diversity is expected to be the same as elsewhere in the genome or even reduced because of background selection and selective sweeps in the non-recombining region.

\section{Population structure}

Similar levels of DNA polymorphism in the recombining and non-recombining loci may perhaps be because of population structure, inflating intraspecific DNA polymorphism in the non-recombining regions due to divergence between populations (Charlesworth et al., 1997; Pannell and Charlesworth, 2000). It is evident from Figure 1 that geographically close samples cluster together in the gene trees, suggesting that the phylogenies based on A1- and A2-linked loci reflect geographic structuring of $M$. violaceum populations. However, these phylogenies are based on the non-recombining loci from the mating-type-linked loci, and the recombining loci may not reveal the same geographic structure. We used Bayesian clustering of samples as implemented in the software Structure2 (Pritchard et al., 2000) to analyse the signal of population structure present in the recombining loci. The posterior probability of the data, $\mathrm{LnP}(\mathrm{D})$, given the number of clusters $(K)$, which is often used as a criterion of the true number of clusters in the data, was highest at $K=7$, but did not form a clear peak (Figure 2a). Instead it plateaus after reaching $K=7$, and the variance between the runs starts increasing. However, if we use the $\Delta K$ criterion (Evanno et al., 2005), the most likely number of clusters is either 2 or 4 , as $\Delta K$ forms two clear peaks (Figure $2 b$ ). The cluster memberships identified by Structure 2 at $K=2,4$ and 7 are shown in Figure 3. With $K=2$, one cluster is composed of Northern and Eastern European samples, whereas the other includes all other samples collected across Southern and Western Europe. Surprisingly, one of the Iberian samples, sample 920, from Valencia appears closer to the Eastern European samples, whereas the other Iberian sample (796 from Galicia) is closer to the Western samples. With $K=4$, the East/West subdivision remains (groups $3 / 1+2$ ), but the two Iberian samples fall into a cluster of their own (group 4) and the Sardinian and Swiss samples (group 1) split up from the rest of the Western European cluster (group 2). With seven clusters, the picture is more complicated and difficult to interpret (Figure 3c). Given that there is no support for $K=7$ using $\Delta K$ and $K=2$ has much lower posterior probability of the 

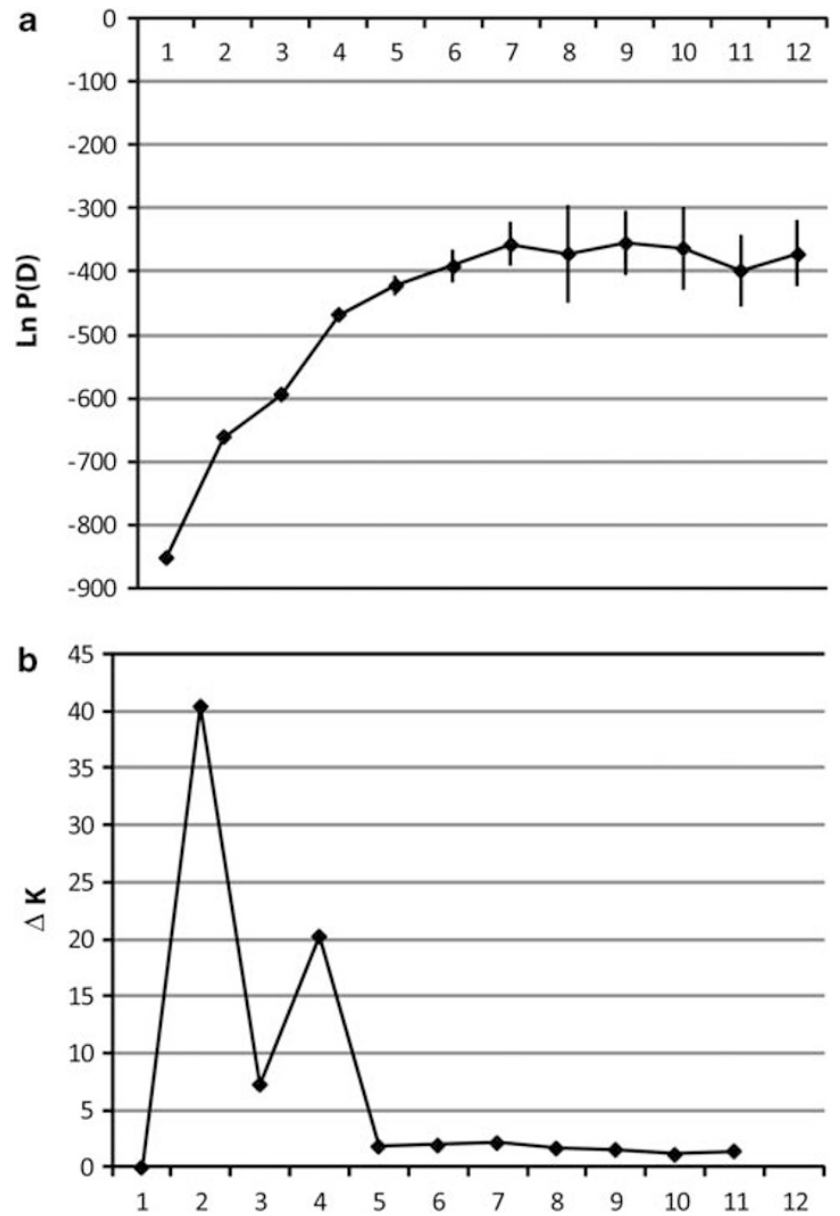

Figure 2 The results of Bayesian clustering analysis of recombining genes using Structure2 (Pritchard et al., 2000). The horizontal axis shows the number of clusters $(K)$. (a) The posterior probability of the data, given the number of clusters, averaged across 10 runs with s.d. between runs. (b) The second order rate of change of the likelihood function $(\Delta K)$ with respect to $K$ (Evanno et al., 2005).

data than $K=4$, four clusters seem to be the optimal number that reflects the population structure present in our sample.

To test whether the population structure affects the comparisons of DNA polymorphism between the recombining and non-recombining regions of the $M$. violaceum genome, we compared the diversity in these regions within the groups identified by Structure analysis. As the clustering of the samples was done with recombining loci only, this approach could underestimate the DNA polymorphism in the recombining regions, and thus the comparison between recombining and non-recombining regions is conservative, if used to test whether the non-recombining regions lack DNA polymorphism compared with recombining regions. This intra-population analysis revealed that the nonrecombining A1- and A2-linked loci contain less polymorphism than the recombining loci (Figure 4). However, once the two-fold difference in ploidy between the mating-type-specific and non-mating-type-specific loci is taken into account, this difference in DNA polymorphism is not significant (HKA test, $P>0.05$ ).

In structured populations, background selection reduces the DNA polymorphism within populations, a
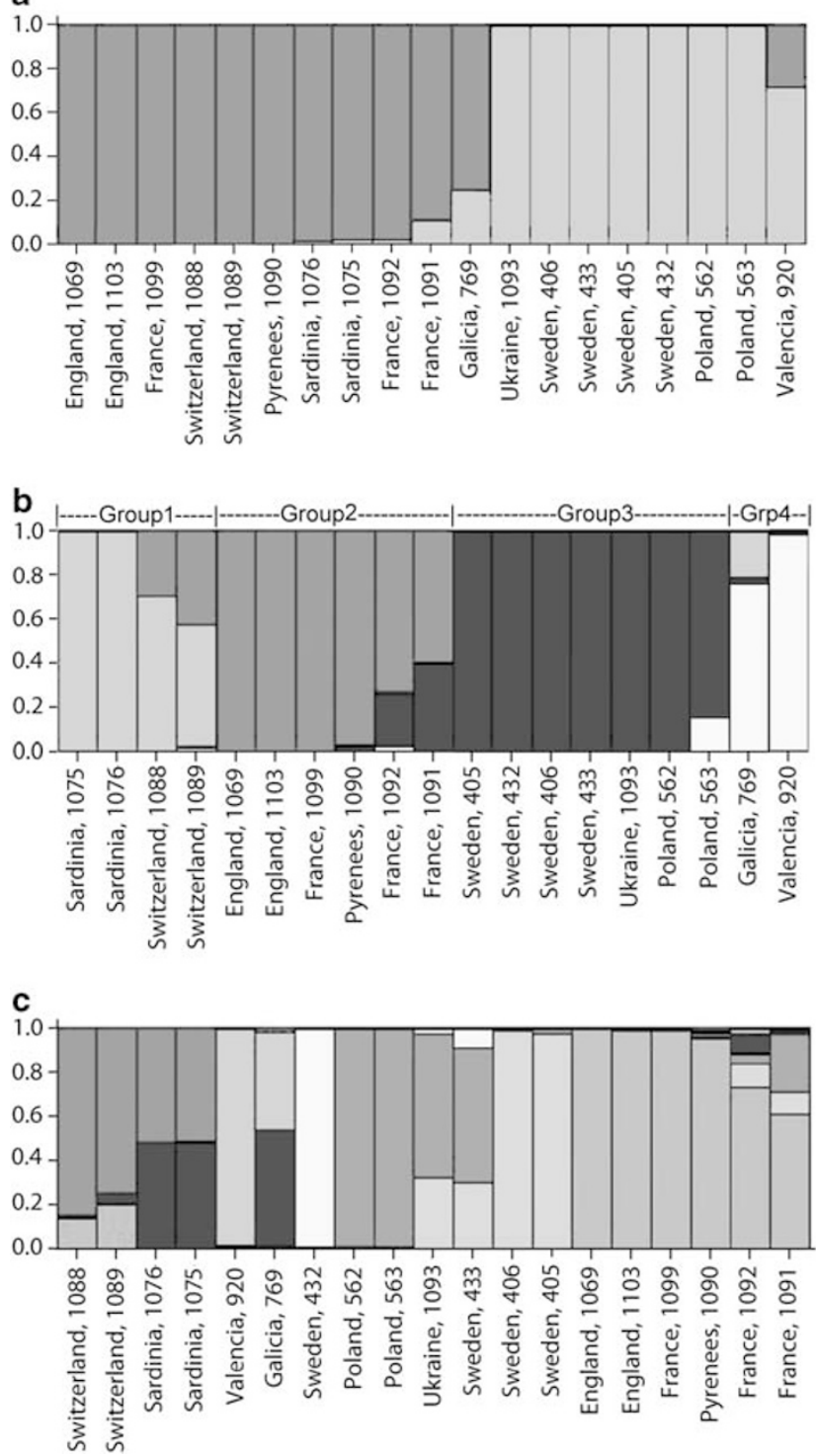

Figure 3 Cluster membership for $K=2$ (a), 4 (b) and 7 (c) based on the recombining regions in the data set, identified by Structure (Pritchard et al., 2000).

but does not reduce between population differentiation (Charlesworth et al., 1997), leading to stronger population subdivision in non-recombining regions (for example, Ironside and Filatov, 2005)). This could negate the difference in the overall DNA polymorphism between recombining and non-recombining regions. Local (intra-population) selective sweeps are expected to have a similar effect, whereas global sweeps (across the species range) should lead to a loss of genetic diversity and population structure preferentially in non-recombining regions. Thus, the presence of population subdivision argues against global sweeps in the nonrecombining mating-type-specific regions of $M$. violaceum.

\section{DNA polymorphism and selfing}

The high degree of selfing in M. violaceum (Giraud et al., 2005) is another factor that may affect the overall level of DNA polymorphism across the genome of this species. In accordance with published microsatellite-based results (Bucheli et al., 2001; Giraud, 2004; Granberg 


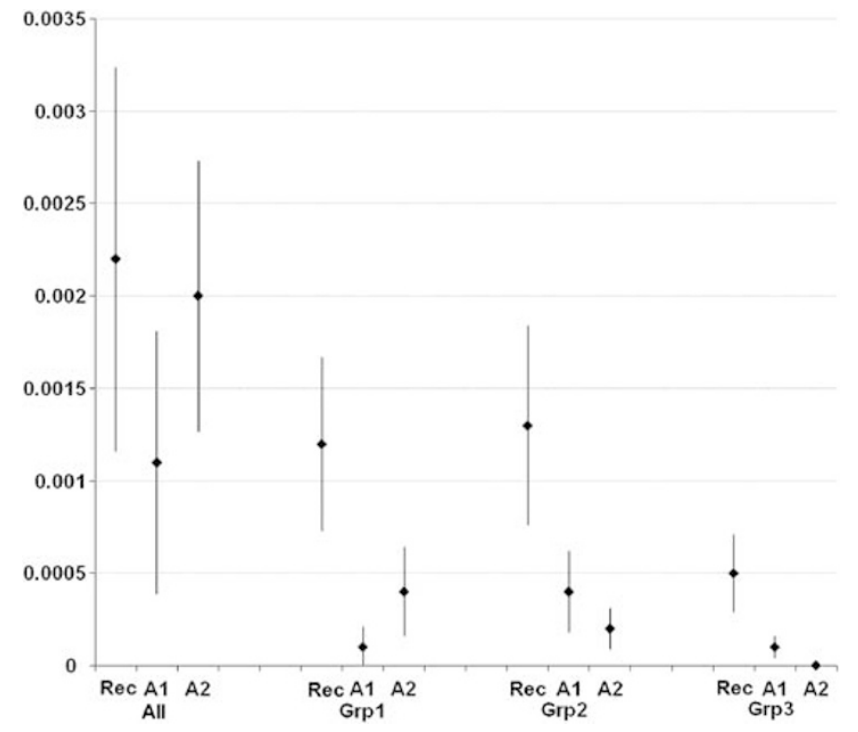

Figure 4 Per-nucleotide DNA polymorphism $(\pi)$ in recombining (Rec) and non-recombining (A1 and A2) regions of the M. violaceum genome for the entire sample (three leftmost data points) and for three sub-populations (groups 1-3, as defined in Figure 3b). Group 4 consists only of two Iberian individuals and is not shown. The error bars represent s.d. of the DNA diversity estimates. et al., 2008), we observed a very low proportion of heterozygotes in our sequence data in $M$. violaceum: only two out of the nineteen individuals in our study had any heterozygous sites in any of the non-mating-type-linked loci studied. A high degree of selfing inflates homozygosity, which leads to a low effective recombination rate in the recombining regions and reduces the difference between the recombining and non-recombining regions. Although for most loci in the recombining regions in our data set, the minimum number of recombination events (Hudson and Kaplan, 1985) is greater than zero, the overall effective recombination rate is still low (Table 3). There is significant linkage disequilibrium (LD) within as well as between most loci in the data set (Figure 5), suggesting that LD in M. violaceum genome extends for megabases, which is similar to LD in Caenorhabditis elegans (Rockman and Kruglyak, 2009), but much wider than in other studied selfers, such as Arabidopsis thaliana (Kim et al., 2007). Genetic mapping in $M$. violaceum revealed crossing-over rates of 0-15\% (Garber et al., 1987; Votintseva and Filatov, 2009). Thus, substantial LD in M. violaceum genome is probably due to high degree of homozygosity in this selfing species rather than to lack of crossing over.

Although selfing reduces the effective recombination rate (via increased homozygosity), LD is still at least

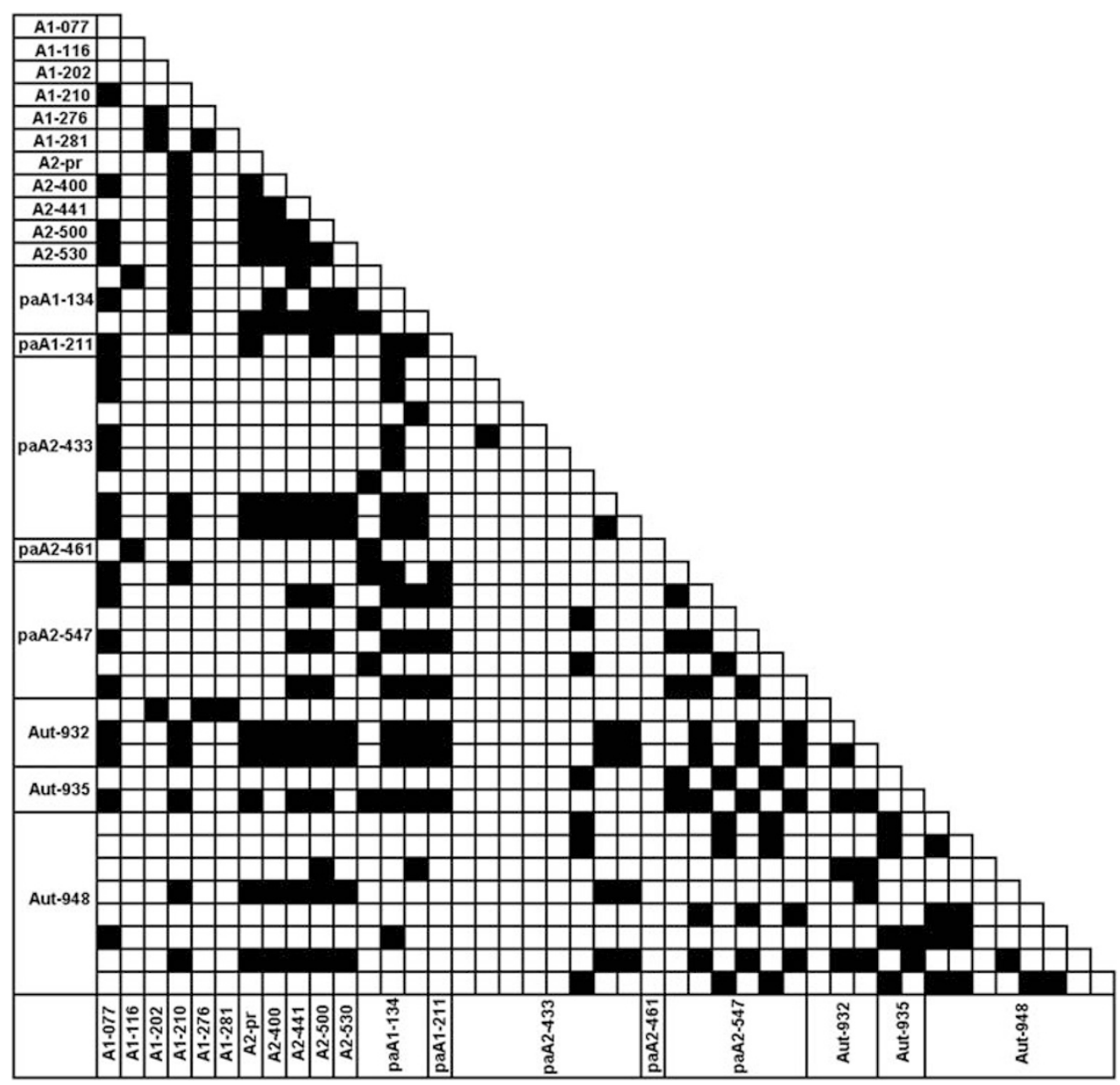

Figure 5 LD between polymorphisms in the studied genes. Significant pairwise LD values $\left(\chi^{2}\right.$-test: $\left.P<0.05 \%\right)$ are shown as black boxes. As polymorphisms in the non-recombining regions are linked, only one randomly chosen polymorphic site is shown for the A1- and A2-linked loci to save space. For the recombining (pa- and aut-) loci, all polymorphisms are shown. 
twice as high in the non-recombining A1- and A2-linked loci as in the recombining regions when measured by the ZnS statistic (Kelly, 1997); see Table 3. Thus, nonrecombining regions might be more prone to various forms of hitchhiking, compared with the rest of the genome despite the selfing-related lack of recombination in the recombining regions. However, the extent to which a region is affected by hitchhiking is proportional not only to LD but also to the number of functional genes and other targets of selection that are linked together. Although LD is higher in non-recombining regions, these regions are relatively small-about $1 \mathrm{Mb}$ (Votintseva and Filatov, 2009) — and may not contain many genes. On the other hand, although LD in recombining regions of $M$. violaceum is lower than in A1- and A2-linked loci, the number of genes 'linked' together by LD is large, as LD extends over the most of the genome. Thus, the genes in the non-recombining regions may not be much affected by genetic hitchhiking (background selection and sweeps) than genes elsewhere in the genome.

\section{Conclusion}

Our comparison of DNA polymorphism in recombining and non-recombining mating-type-specific regions of M. violaceum revealed relatively low polymorphism in all loci and no evidence for a significant reduction of polymorphism in non-recombining regions. We also observed significant population structuring and a high level of homozygosity in our data set, as expected in a predominantly selfing species. We conclude that the selfing-related reduction of recombination across the $M$. violaceum genome negates the difference in the level of DNA polymorphism between the recombining and non-recombining regions. This conclusion is supported by extensive LD. Thus, genetic degeneration due to a lack of recombination in $M$. violaceum may not be restricted to non-recombining regions of its 'sex chromosomes', and genes in this species might undergo similar genetic degeneration in the mating-type-specific regions and elsewhere in the genome. It will be possible to test this hypothesis as soon as the sequence of the $M$. violaceum genome will become available.

\section{Conflict of interest}

The authors declare no conflict of interest.

\section{Acknowledgements}

We are grateful to Chris Dixon for corrections of the manuscript and to the BBSRC and John Fell fund (Oxford) for funding.

\section{References}

Bucheli E, Gautschi B, Shykoff JA (2001). Differences in population structure of the anther smut fungus Microbotryum violaceum on two closely related host species, Silene latifolia and S dioica. Mol Ecol 10: 285-294.

Charlesworth B, Morgan MT, Charlesworth D (1993). The effect of deleterious mutations on neutral molecular variation. Genetics 134: 1289-1303.

Charlesworth B, Nordborg M, Charlesworth D (1997). The effects of local selection, balanced polymorphism and background selection on equilibrium patterns of genetic diversity in subdivided populations. Genet Res 70: 155-174.
Charlesworth D (2006). Balancing selection and its effects on sequences in nearby genome regions. PLoS Genet 2: e64.

Charlesworth D, Charlesworth B (2005). Sex chromosomes: evolution of the weird and wonderful. Curr Biol 15: R129-R131.

Charlesworth D, Charlesworth B, Morgan MT (1995). The pattern of neutral molecular variation under the background selection model. Genetics 141: 1619-1632.

De Vienne DM, Refregier G, Hood ME, Guigue A, Devier B, Vercken $E$ et al. (2009). Hybrid sterility and inviability in the parasitic fungal species complex Microbotryum. J Evol Biol 22: 683-698.

Denchev CM, Giraud T, Hood ME (2009). Three new species of anthericolous smut fungi on Caryophyllaceae. Mycologica Balcanica 6: 79-84.

Evanno G, Regnaut S, Goudet J (2005). Detecting the number of clusters of individuals using the software STRUCTURE: a simulation study. Mol Ecol 14: 2611-2620.

Felsenstein J (1974). The evolutionary advantage of recombination. Genetics 78: 737-756.

Filatov DA (2009). Processing and population genetic analysis of multigenic datasets with ProSeq3 software. Bioinformatics 25: 3189-3190.

Filatov DA, Moneger F, Negrutiu I, Charlesworth D (2000). Low variability in a Y-linked plant gene and its implications for Y-chromosome evolution. Nature 404: 388-390.

Fraser JA, Diezmann S, Subaran RL, Allen A, Lengeler KB, Dietrich FS et al. (2004). Convergent evolution of chromosomal sex-determining regions in the animal and fungal kingdoms. PLoS Biol 2: e384.

Garber ED, Eng C, Stevens DM (1987). Genetics of Ustilago violacea. XXI. Centromere-linkage values and pericentric gene clustering. 12: 555-560.

Gaut BS, Wright SI, Rizzon C, Dvorak J, Anderson LK (2007). Recombination: an underappreciated factor in the evolution of plant genomes. Nat Rev Genet 8: 77-84.

Giraud T (2004). Patterns of within population dispersal and mating of the fungus Microbotryum violaceum parasitising the plant Silene latifolia. Heredity 93: 559-565.

Giraud T, Jonot O, Shykoff JA (2005). Selfing propensity under choice conditions in a parasitic fungus, Microbotryum violaceum, and parameters influencing infection success in artificial inoculations. Int I Plant Sci 166: 649-657.

Giraud T, Yockteng R, Lopez-Villavicencio M, Refregier G, Hood ME (2008). Mating system of the anther smut fungus Microbotryum violaceum: selfing under heterothallism. Eukaryot Cell 7: 765-775.

Glass NL, Vollmer SJ, Staben C, Grotelueschen J, Metzenberg RL, Yanofsky C (1988). DNAs of the two mating-type alleles of Neurospora crassa are highly dissimilar. Science 241: 570-573.

Granberg A, Carlsson-Graner U, Arnqvist P, Giles B (2008) Variation in breeding system traits within and among populations of Microbotryum violaceum on Silene dioica. Int J Plant Sci 169: 293-303.

Hill WG, Robertson A (1966). The effect of linkage on limits to artificial selection. Genet Res 8: 269-294.

Hood ME (2002). Dimorphic mating-type chromosomes in the fungus Microbotryum violaceum. Genetics 160: 457-461.

Hood ME, Antonovics J (2004). Mating within the meiotic tetrad and the maintenance of genomic heterozygosity. Genetics 166: 1751-1759.

Hood ME, Antonovics J, Koskella B (2004). Shared forces of sex chromosome evolution in haploid-mating and diploid-mating organisms: Microbotryum violaceum and other model organisms. Genetics 168: 141-146.

Hudson RR (1987). Estimating the recombination parameter of a finite population model without selection. Genet Res $\mathbf{5 0}$ 245-250.

Hudson RR (2002). Generating samples under a Wright-Fisher neutral model of genetic variation. Bioinformatics 18: 337-338. 
Hudson RR, Kaplan NL (1985). Statistical properties of the number of recombination events in the history of a sample of DNA sequences. Genetics 111: 147-164.

Hudson RR, Kreitman M, Aguade M (1987). A test of neutral molecular evolution based on nucleotide data. Genetics 116: 153-159.

Ironside JE, Filatov DA (2005). Extreme population structure and high interspecific divergence of the Silene $\mathrm{Y}$ chromosome. Genetics 171: 705-713.

James TY, Liou SR, Vilgalys R (2004). The genetic structure and diversity of the $\mathrm{A}$ and $\mathrm{B}$ mating-type genes from the tropical oyster mushroom, Pleurotus djamor. Fungal Genet Biol 41: 813-825.

Kelly JK (1997). A test of neutrality based on interlocus associations. Genetics 146: 1197-1206.

Kim S, Plagnol V, Hu TT, Toomajian C, Clark RM, Ossowski S et al. (2007). Recombination and linkage disequilibrium in Arabidopsis thaliana. Nat Genet 39: 1151-1155.

Larracuente AM, Sackton TB, Greenberg AJ, Wong A, Singh ND, Sturgill D et al. (2008). Evolution of protein-coding genes in Drosophila. Trends Genet 24: 114-123.

Le Gac M, Hood ME, Fournier E, Giraud T (2007). Phylogenetic evidence of host-specific cryptic species in the anther smut fungus. Evolution 61: 15-26.

Lengeler KB, Fox DS, Fraser JA, Allen A, Forrester K, Dietrich FS et al. (2002). Mating-type locus of Cryptococcus neoformans: a step in the evolution of sex chromosomes. Eukaryot Cell 1: 704-718.

Lewontin R (1964). The interaction of selection and linkage. I. General considerations: heterotic models. Genetics 49: 49-67.

Librado P, Rozas J (2009). DnaSP v5: a software for comprehensive analysis of DNA polymorphism data. Bioinformatics 25: 1451-1452.

Maynard Smith J, Haigh J (1974). The hitchhiking effect of a favourable gene. 23: 23-35.

Menkis A, Jacobson DJ, Gustafsson T, Johannesson H (2008). The mating-type chromosome in the filamentous ascomycete Neurospora tetrasperma represents a model for early evolution of sex chromosomes. PLoS Genet 4: e1000030.

Pannell JR, Charlesworth B (2000). Effects of metapopulation processes on measures of genetic diversity. Philos Trans $R$ Soc Lond B Biol Sci 355: 1851-1864.
Pritchard JK, Stephens M, Donnelly P (2000). Inference of population structure using multilocus genotype data. Genetics 155: 945-959.

Rice WR (1987). Genetic hitchhiking and the evolution of reduced genetic activity of the $\mathrm{Y}$ sex chromosome. Genetics 116: 161-167.

Rockman MV, Kruglyak L (2009). Recombinational landscape and population genomics of Caenorhabditis elegans. PLoS Genet 5: e1000419.

Ruddat M., Kokontis J, Birch L, Garber ED, Chiang K-S, Campanella J et al. (1991). Interactions of Microbotryum violaceum (Ustilago violacea) with its host plant Silene alba. Plant Science 80: 157-165.

Scheet P, Stephens M (2006). A fast and flexible statistical model for large-scale population genotype data: applications to inferring missing genotypes and haplotypic phase. Am J Hum Genet 78: 629-644.

Tajima F (1983). Evolutionary relationship of DNA sequences in finite populations. Genetics 105: 437-460.

Tajima F (1989). Statistical method for testing the neutral mutation hypothesis by DNA polymorphism. Genetics 123: 585-595.

Tamura K, Dudley J, Nei M, Kumar S (2007). MEGA4: Molecular Evolutionary Genetics Analysis (MEGA) software version 4.0. Mol Biol Evol 24: 1596-1599.

Uyenoyama MK (2005). Evolution under tight linkage to mating type. New Phytol 165: 63-70.

Votintseva AA, Filatov DA (2009). Evolutionary strata in a small mating-type-specific region of the smut fungus Microbotryum violaceum. Genetics 182: 1391-1396.

Watterson G (1975). On the number of segregating sites in genetic models without recombination. Theor Popul Biol 7: 256-276.

Yi S, Charlesworth B (2000). Contrasting patterns of molecular evolution of the genes on the new and old sex chromosomes of Drosophila miranda. Mol Biol Evol 17: 703-717.

Yockteng R, Marthey S, Chiapello H, Gendrault A, Hood ME, Rodolphe F et al. (2007). Expressed sequences tags of the anther smut fungus, Microbotryum violaceum, identify mating and pathology genes. BMC Genomics 8: 272.

Zambino P, Groth JV, Lukens L, Garton JR, May G (1997). Variation at the $\mathrm{b}$ mating type locus of Ustilago maydis. Phytopathology 87: 1233-1239. 